\title{
Control and Configuration Planning of an Aerial Cable Towed System
}

\author{
Julian Erskine $^{1}$, Abdelhamid Chriette ${ }^{2}$, Stéphane Caro $^{3}$, Member, IEEE
}

\begin{abstract}
This paper investigates the effect of the robot configuration on the performance of an aerial cable towed system (ACTS) composed of three quadrotors manipulating a point mass payload. The kinematic and dynamic models of the ACTS are derived in a minimal set of geometric coordinates, and a centralized feedback linearization controller is developed. Independent to the payload trajectory, the configuration of the ACTS is controlled and is evaluated using a robustness index named the capacity margin. Experimental validation is performed with optimal, suboptimal, and wrench infeasible configurations. It is shown that configurations near the point of zero capacity margin allow the ACTS to hover but not to follow dynamic trajectories, and that the ACTS cannot fly with a negative capacity margin. Dynamic tests are performed on the ACTS, showing the effects of the configuration on the achievable accelerations.
\end{abstract}

Keywords: Aerial Systems, Multi-Robot Systems, Quadrotors, Control, Reconfiguration, Cable-Driven Parallel Robots

\section{INTRODUCTION}

Unmanned aerial vehicles (UAVs) have become increasingly used in commercial applications in recent years due to the miniaturization of computing technology. Quadrotors in particular have proven commercial applications in entertainment and surveillance applications, and much work is currently underway towards adapting their use to construction, inspection, and logistics industries. They have the benefit of being lightweight, relatively inexpensive, and of simple design compared to other UAVs. Moreover, quadrotors are agile, can hover, and have vertical takeoff and landing capabilities [1]. The primary drawback of quadrotors is the coupling between their rotational and translational dynamics, their limited payload and range due to their small size. It is therefore of great interest to study collaborative manipulation between multiple quadrotors to increase the payload, and allow for decoupling of the payload's rotational and translational motions. Many solutions use rigid links, flexible links, or cables to connect the quadrotors and payload [2], [3].

Studies of aerial cable towed systems (ACTSs) have focused primarily on designing controllers to stabilize the payload and quadrotors using a variety of methods, both in quasi-static and dynamic scenarios. In [4], [5], the control is achieved using a linear quadratic regulator (LQR). In [6], ACTSs are proven to be differentially flat, and a dynamic control based on differentially flat trajectories

\footnotetext{
${ }^{1}$ École Centrale de Nantes (ECN), Laboratoire des Sciences du Numérique de Nantes (LS2N), UMR CNRS 6004, 1 rue de la Noe, 44321 Nantes, France. julian.erskinedls2n. fr

2 ECN, LS2N. abdelhamid. chrietteels2n. fr

3 LS2N, Centre National de la Recherche Scientifique (CNRS), France. stephane.carodls2n.fr
}

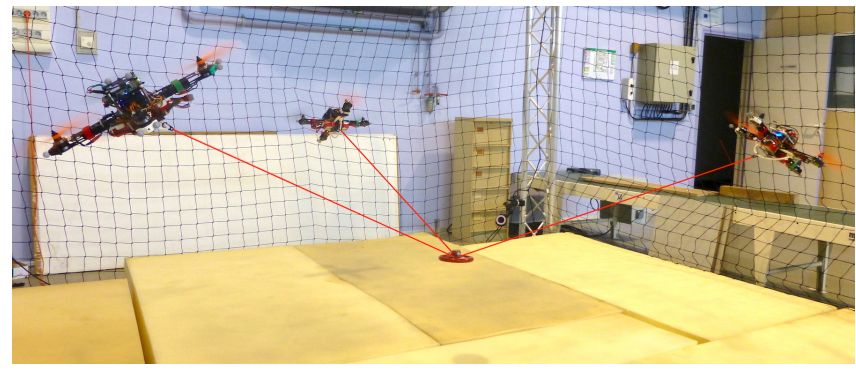

Fig. 1: ACTS prototype developed at Centrale Nantes, LS2N

is demonstrated, but requires the sixth derivative of the payload position and fourth derivative of the cable tensions. A geometric controller is proposed and simulated in [7], with the configuration expressed in the local coordinates of each quadrotor to avoid representation singularities. These methodologies are based on a centralized controller with knowledge of payload and quadrotor poses. A controller proposed in [8] is based on graph theory and uses only local information available to each quadrotor. The work in [9] and [10] moves towards distributed control of ACTS with two quadrotors for deployment in more realistic situations.

In [11], a centralized controller was developed by considering the ACTS as a continuously reconfigurable cabledriven parallel robot (CDPR). This has the advantage of allowing the use of well established control techniques from the field of parallel robotics. A feedback linearization controller is proposed and simulated for an over-actuated ACTS, however the relative position of each quadrotor with respect to the platform (the configuration) was arbitrarily chosen. Expanding on the idea of ACTSs as continuously reconfigurable CDPRs, previous work in [12] extended a wrench analysis method introduced in [13] and used in the field of CDPRs to account for quadrotor actuation constraints. The degree of inclusion of the task wrench within the available wrench set was introduced in [14] and is called the capacity margin. This robustness index is used in [15] as an objective function in the design and discrete reconfiguration planning of CDPRs.

This paper presents a modified version of a feedback linearization controller, also called a computed torque controller in robotics, for an ACTS composed of three quadrotors and a point mass end-effector. The control inputs are the payload position and the configuration parameters of the ACTS, expressed using spherical coordinates. The ACTS configuration is varied to test the capacity margin as a function of different payload masses, to assess its use as a robustness index. The paper is organized as follows: 
Section II deals with the kinematic and dynamic modelling of the ACTS. Section III explains the control architecture of the system, and the experimental methodology is detailed in Sec. IV. Section V is about the procedure for choosing the configuration, and the experimental results are presented in Sec. VI. Conclusions and future work are drawn out in Sec. VII.

\section{MODELLING}

Modelling of an ACTS can be divided into three primary components: $(i)$ the actuation and dynamics of the individual quadrotors; (ii) the kinematic relationship between the quadrotors and the payload; and (iii) the dynamic relationship between the forces acting on the quadrotor and those acting on the payload. The parameterization of the ACTS is shown in Fig. 2, with a base frame $\mathcal{F}_{0}$ of origin $O$ and axes $x_{0}, y_{0}$ and $z_{0}$. The payload $P$ is described by the Cartesian coordinate vector $\mathbf{x}_{p}$ expressed in frame $\mathcal{F}_{0}$. Frame $\mathcal{F}_{i}$, of origin $O_{i}$ and axes $x_{i}, y_{i}$ and $z_{i}$, is attached to the geometric center of the $i$ th quadrotor while $\mathbf{R}_{i}$ is the rotation matrix from $\mathcal{F}_{0}$ to $\mathcal{F}_{i}$. Each quadrotor is connected to the payload by a massless and therefore straight cable of length $l_{i}$ and tension $t_{i},\left(t_{i}>0\right)$ along unit vector $\mathbf{u}_{i}=\overrightarrow{P O_{i}} / l_{i}$. The gravity vector is $\mathbf{g}=\left[\begin{array}{lll}0 & 0 & -9.81\end{array}\right]^{T} \mathrm{~ms}^{-2}$.

\section{A. Modelling of Quadrotors}

Quadrotors are a class of UAV with four co-planar propellors, where each propellor generates a thrust force $f=C_{T} \omega^{2}$ and a drag moment $m=C_{D} \omega^{2}$, in which $C_{T}$ and $C_{D}$ are the coefficients of thrust and drag respectively, and $\omega$ is the angular velocity of the propellor. The four propellors together exert a force $f_{z}$ normal to the plane of the propellors and three independent moments $m_{x}, m_{y}$, and $m_{z}$ about $O_{i}$ given by (1) expressed in $\mathcal{F}_{i}$, with $\boldsymbol{\omega}_{a c t}=\left[\begin{array}{llll}\omega_{1}^{2} & \omega_{2}^{2} & \omega_{3}^{2} & \omega_{4}^{2}\end{array}\right]^{T}$ being the vector of actuation variables of the quadrotor.

$$
\left[\begin{array}{c}
f_{z} \\
m_{x} \\
m_{y} \\
m_{z}
\end{array}\right]=\left[\begin{array}{cccc}
C_{T} & C_{T} & C_{T} & C_{T} \\
-r C_{T} & 0 & r C_{T} & 0 \\
0 & -r C_{T} & 0 & r C_{T} \\
-C_{D} & C_{D} & -C_{D} & C_{D}
\end{array}\right]\left[\begin{array}{c}
\omega_{1}^{2} \\
\omega_{2}^{2} \\
\omega_{3}^{2} \\
\omega_{4}^{2}
\end{array}\right]
$$

The thrust $\mathbf{f}_{i}$ of the $i^{\text {th }}$ quadrotor with magnitude $f_{i}$ along unit vector $\mathbf{v}_{i}$ in the world frame is $\mathbf{f}_{i}=\mathbf{R}_{i}\left[\begin{array}{ccc}0 & 0 & f_{z}\end{array}\right]^{T}$, and its moment vector is $\mathbf{m}_{i}=\mathbf{R}_{i}\left[\begin{array}{lll}m_{x} & m_{y} & m_{z}\end{array}\right]^{T}$. The dynamic model of the $i^{\text {th }}$ quadrotor is expressed by (2) and (3) in $\mathcal{F}_{0}$, showing a decoupling between rotational and translational dynamics.

$$
\begin{aligned}
f_{i} \mathbf{v}_{i}+m_{i} \mathbf{g}-t_{i} \mathbf{u}_{i} & =m_{i} \ddot{\mathbf{x}}_{i} \\
\mathbf{m}_{i} & =\mathbb{J}_{i} \dot{\boldsymbol{\omega}}_{i}+\boldsymbol{\omega}_{i} \times \mathbb{J}_{i} \boldsymbol{\omega}_{i}
\end{aligned}
$$

where $m_{i}$ is the mass of the $i^{\text {th }}$ quadrotor, $\boldsymbol{\omega}_{i}$ is its angular velocity vector, and $\mathbb{J}_{i}$ is its inertia matrix.

\section{B. Kinematic Modelling of the ACTS}

The ACTS is being modelled as a traditional parallel robot, however unlike CDPRs where the cable length $l_{i}$ is actuated, the position of the cable endpoint $\mathbf{x}_{i}=\overrightarrow{O O}_{i}$ is actuated,

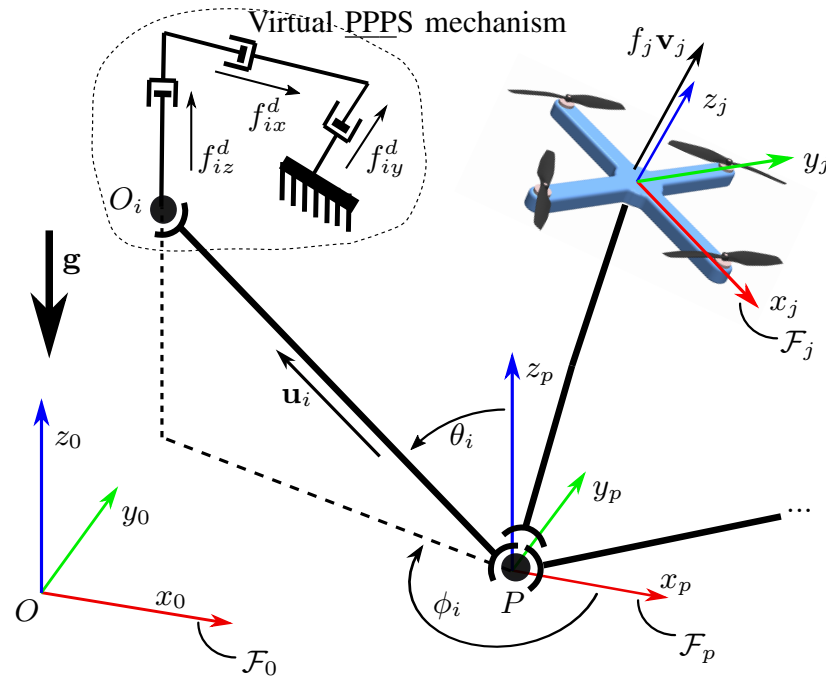

Fig. 2: Parameterization of the ACTS showing the structure of the virtual parallel mechanism.

similar to a $\underline{\mathrm{PPP}}^{1}$ translational mechanism. As the cable is unable to support moments and is always in tension, it can be modelled as a rigid link with a spherical joint at each end, assuming that $t>0 N$. The robot can therefore be considered as a 3-PPPSS mechanism.

The loop closure equation for the limb $i$ is given by (4), with $\mathbf{x}_{p}$ being the cartesian coordinate vector of the payload $\overrightarrow{O P}$ expressed in $\mathcal{F}_{0}$.

$$
\mathbf{x}_{i}=\mathbf{x}_{p}+l_{i} \mathbf{u}_{i}
$$

Using ISO spherical coordinates where the reference frame $\mathcal{F}_{p}$ is a translation of $\mathcal{F}_{0}$ by $\mathbf{x}_{p}$, the cable vector is expressed as

$$
\mathbf{u}_{i}=\left[\begin{array}{c}
\mathrm{c} \phi_{i} \mathrm{~s} \theta_{i} \\
\mathrm{~s} \phi_{i} \mathrm{~s} \theta_{i} \\
\mathrm{c} \theta_{i}
\end{array}\right]
$$

where $\mathrm{c} x$ and $\mathrm{s} x$ represent $\cos (x)$ and $\sin (x)$ respectively. The azimuth angle $\phi_{i}$ and inclination angle $\theta_{i}$ are:

$$
\begin{aligned}
\theta_{i} & =\cos ^{-1}\left(\frac{x_{i, z}-x_{p, z}}{l_{i}}\right) \\
\phi_{i} & =\operatorname{atan} 2\left(x_{i, y}-x_{p, y}, x_{i, x}-x_{p, x}\right)
\end{aligned}
$$

The task space state vector of the ACTS is $\mathbf{X}=\left[\begin{array}{ll}\mathbf{x}_{p}^{T} & \mathcal{C}^{T}\end{array}\right]^{T}$ in which the configuration vector is $\mathcal{C}=\left[\begin{array}{llllll}\phi_{1} & \theta_{1} & \phi_{2} & \theta_{2} & \phi_{3} & \theta_{3}\end{array}\right]^{T}$. The first-order direct kinematic model (DKM) relates the quadrotor velocities to the state derivatives, through the form $\dot{\mathbf{X}}=\mathbf{J}\left[\begin{array}{lll}\dot{\mathbf{x}}_{1}^{T} & \dot{\mathbf{x}}_{2}^{T} & \dot{\mathbf{x}}_{3}^{T}\end{array}\right]^{T}$, where $\mathbf{J}$ is the kinematic jacobian matrix. This is done by differentiating (4) with respect to time giving (7).

$$
\dot{\mathbf{x}}_{i}=\dot{\mathbf{x}}_{p}+l_{i} \dot{\mathbf{u}}_{i}
$$

${ }^{1} \mathrm{P}$ stands for a prismatic joint, $\mathrm{S}$ stands for a spherical joint. An underlined letter means that the corresponding joint is actuated. 
The DKM of the ACTS is therefore expressed in (8) as:

$$
\dot{\mathbf{X}}=\mathbf{J}\left[\begin{array}{c}
\dot{\mathbf{x}}_{1} \\
\dot{\mathbf{x}}_{2} \\
\dot{\mathbf{x}}_{3}
\end{array}\right] \text {, where } \mathbf{J}=\left[\begin{array}{cccc}
\mathbb{I}_{3} & \mathbf{C}_{1} & \mathbf{0} & \mathbf{0} \\
\mathbb{I}_{3} & \mathbf{0} & \mathbf{C}_{2} & \mathbf{0} \\
\mathbb{I}_{3} & \mathbf{0} & \mathbf{0} & \mathbf{C}_{3}
\end{array}\right]^{-1}
$$

with $\mathbb{I}_{3}$ being the identity matrix of rank 3 , and $\mathbf{C}_{i}$ being the matrix defined in (9).

$$
\mathbf{C}_{i}=l_{i}\left[\begin{array}{cc}
-\mathrm{s} \phi_{i} s \theta_{i} & \mathrm{c} \phi_{i} \mathrm{c} \theta_{i} \\
\mathrm{c} \phi_{i} s \theta_{i} & \mathrm{~s} \phi_{i} \mathrm{c} \theta_{i} \\
0 & -\mathrm{s} \theta_{i}
\end{array}\right]
$$

To determine the dynamic model of the ACTS in Sec. II-C, the second-order DKM (10) must be derived from a differentiation of (7).

$$
\ddot{\mathbf{X}}=\mathbf{J}\left[\begin{array}{lll}
\ddot{\mathbf{x}}_{1}^{T} & \ddot{\mathbf{x}}_{2}^{T} & \ddot{\mathbf{x}}_{3}^{T}
\end{array}\right]^{T}+\mathbf{b}
$$

where the non-linear component is $\mathbf{b}=\mathbf{J}\left[\begin{array}{lll}\mathbf{b}_{1}^{T} & \mathbf{b}_{2}^{T} & \mathbf{b}_{3}^{T}\end{array}\right]^{T}$ in which $\mathbf{b}_{i}=-l_{i}\left[\begin{array}{ccc}\mathrm{c} \phi_{i} \mathrm{~s} \theta_{i} & \mathrm{c} \phi_{i} \mathrm{~s} \theta_{i} & 2 \mathrm{~s} \phi_{i} \mathrm{c} \theta_{i} \\ \mathrm{~s} \phi_{i} \mathrm{~s} \theta_{i} & \mathrm{~s} \phi_{i} \mathrm{~s} \theta_{i} & 0 \\ 0 & \mathrm{c} \theta_{i} & 0\end{array}\right]\left[\begin{array}{c}\dot{\phi}_{i}^{2} \\ \dot{\theta}_{i}^{2} \\ \dot{\phi}_{i} \dot{\theta}_{i}\end{array}\right]$

\section{Dynamic Modelling of the ACTS}

The forces acting on the payload are described by (11), where $m_{p}$ is the mass of the payload.

$$
m_{p} \mathbf{g}+\sum_{j=1}^{3}\left(t_{j} \mathbf{u}_{j}\right)+\mathbf{w}_{e}=m_{p} \ddot{\mathbf{x}}_{p}
$$

The cable tension vector $\mathbf{t}=\left[\begin{array}{lll}t_{1} & t_{2} & t_{3}\end{array}\right]^{T}$ can be computed through the inversion of the wrench matrix $\mathbf{W}=\left[\begin{array}{lll}\mathbf{u}_{1} & \mathbf{u}_{2} & \mathbf{u}_{3}\end{array}\right]$, as shown in (12) so long as $\mathbf{W}$ is not singular.

$$
\mathbf{t}=-\mathbf{W}^{-1}\left(m_{p} \mathbf{g}+\mathbf{w}_{e}\right)
$$

where $\mathbf{w}_{e}$ is an external wrench (purely a force in point mass cases) acting on the payload. For this model to be defined, $\mathbf{W}$ must be invertable, therefore $\mathbf{u}_{1}, \mathbf{u}_{2}$, and $\mathbf{u}_{3}$ must not be co-planar.

Equations (10) and (12) are substituted into (2) to express the resultant thrust vector of each quadrotor for a given state $(\mathbf{X}, \dot{\mathbf{X}}, \ddot{\mathbf{X}})$. Putting this equation in the standard inverse dynamic model (IDM) form $\mathbf{f}=\mathbf{D} \ddot{\mathbf{X}}+\mathbf{G}$ results in (13), where $\mathbf{f}=\left[\begin{array}{lll}\mathbf{f}_{1}{ }^{T} & \mathbf{f}_{2}{ }^{T} & \mathbf{f}_{3}{ }^{T}\end{array}\right]^{T}$ contains the actuation forces of the three quadrotors.

$$
\mathbf{f}=\underbrace{\left(\mathbf{M}_{Q} \mathbf{J}^{-1}+\mathbf{T}_{\ddot{x}_{p}}\right)}_{\mathbf{D}} \ddot{\mathbf{X}}-\underbrace{\mathbf{M}_{Q}\left(\left[\begin{array}{l}
\mathbf{g} \\
\mathbf{g} \\
\mathbf{g}
\end{array}\right]+\mathbf{J}^{-1} \mathbf{b}\right)-\mathbf{T}_{g}}_{\mathbf{G}}
$$

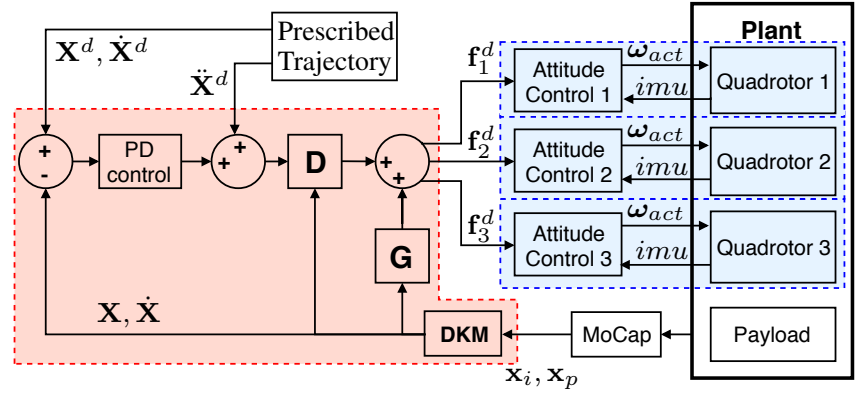

Fig. 3: Control diagram showing the three inner loops (blue), and the outer loop (red). There is a numerical differentiation inside the DKM block, but can be replaced by other estimations such as the Kalmann filtered velocity of each quadrotor.

in which matrices $\mathbf{M}_{Q}, \mathbf{T}_{\ddot{x}_{p}}$, and $\mathbf{T}_{g}$ take the form

$$
\begin{aligned}
\mathbf{M}_{Q}= & {\left[\begin{array}{lll}
m_{1} \mathbb{I}_{3} & \mathbf{0}_{3 \times 3} & \mathbf{0}_{3 \times 3} \\
\mathbf{0}_{3 \times 3} & m_{2} \mathbb{I}_{3} & \mathbf{0}_{3 \times 3} \\
\mathbf{0}_{3 \times 3} & \mathbf{0}_{3 \times 3} & m_{3} \mathbb{I}_{3}
\end{array}\right] } \\
\mathbf{T}_{\ddot{x}_{p}}= & m_{p}\left[\begin{array}{ll}
\mathbf{u}_{1} \boldsymbol{i} \mathbf{W}^{-1}, & \mathbf{0}_{3 \times 6} \\
\mathbf{u}_{2} \boldsymbol{j} \mathbf{W}^{-1}, & \mathbf{0}_{3 \times 6} \\
\mathbf{u}_{3} \boldsymbol{k} \mathbf{W}^{-1}, & \mathbf{0}_{3 \times 6}
\end{array}\right] \\
\mathbf{T}_{g}= & {\left[\begin{array}{l}
\mathbf{u}_{1} \boldsymbol{i} \mathbf{W}^{-1}\left(m_{p} \mathbf{g}+\mathbf{w}_{e}\right) \\
\mathbf{u}_{2} \boldsymbol{j} \mathbf{W}^{-1}\left(m_{p} \mathbf{g}+\mathbf{w}_{e}\right) \\
\mathbf{u}_{3} \boldsymbol{k} \mathbf{W}^{-1}\left(m_{p} \mathbf{g}+\mathbf{w}_{e}\right)
\end{array}\right] }
\end{aligned}
$$

with $\boldsymbol{i}=\left[\begin{array}{lll}1 & 0 & 0\end{array}\right], \boldsymbol{j}=\left[\begin{array}{lll}0 & 1 & 0\end{array}\right]$, and $\boldsymbol{k}=\left[\begin{array}{lll}0 & 0 & 1\end{array}\right]$.

The dynamic model is singular when $\mathbf{W}$ in non-invertable (corresponding to an actuation singularity) or when $\mathbf{J}$ in noninvertible, which occurs when at least two cables are vertical (impossible due to collisions), or when at least two cables are horizontal and have $\phi_{i}=k \pi / 2$ for $k \in \mathbb{Z}$.

\section{CONTROLLER DESIGN}

As is common with quadrotor control, a back-stepping control architecture is used. The centralized outer loop determines the desired thrust vector $\mathbf{f}_{i}^{d}$ of each quadrotors to force the states $(\mathbf{X}, \dot{\mathbf{X}})$ to the desired states $\left(\mathbf{X}^{d}, \dot{\mathbf{X}}^{d}\right)$. The inner loops onboard each quadrotor stabilize the attitude of the quadrotors such that the rotation $\mathbf{R}_{i}$ of the $i^{t h}$ quadrotor converges to satisfy $\mathbf{R}_{i}\left[\begin{array}{lll}0 & 0 & 1\end{array}\right]^{T}=\mathbf{f}_{i}^{d} / f_{i}^{d}$. The global control architecture is shown in Fig. 3. Simulations were performed using co-simulation between Simulink and ADAMS to validate the controller's asymptotic convergence.

\section{A. OUTER CONTROL LOOP}

The outer control loop uses an auxiliary control law composed of a PD controller on the task space error $\mathbf{e}=\mathbf{X}^{d}-\mathbf{X}$, and the desired acceleration $\ddot{\mathbf{X}}^{d}$. The auxiliary control law is multiplied by the matrix of linear components of the dynamic model $\mathbf{D}$, and the vector of non-linear compensation $\mathbf{G}$ is added, the sum of which is the desired force vector $\mathbf{f}^{d}$ of the ACTS, where $\mathbf{f}^{d}=\left[\begin{array}{lll}\mathbf{f}_{1}^{d^{T}} & \mathbf{f}_{2}^{d^{T}} & \mathbf{f}_{3}^{d^{T}}\end{array}\right]^{T}$.

$$
\mathbf{f}^{d}=\mathbf{D}\left(\ddot{\mathbf{X}}^{d}+k_{D} \dot{\mathbf{e}}+k_{P} \mathbf{e}\right)+\mathbf{G}
$$



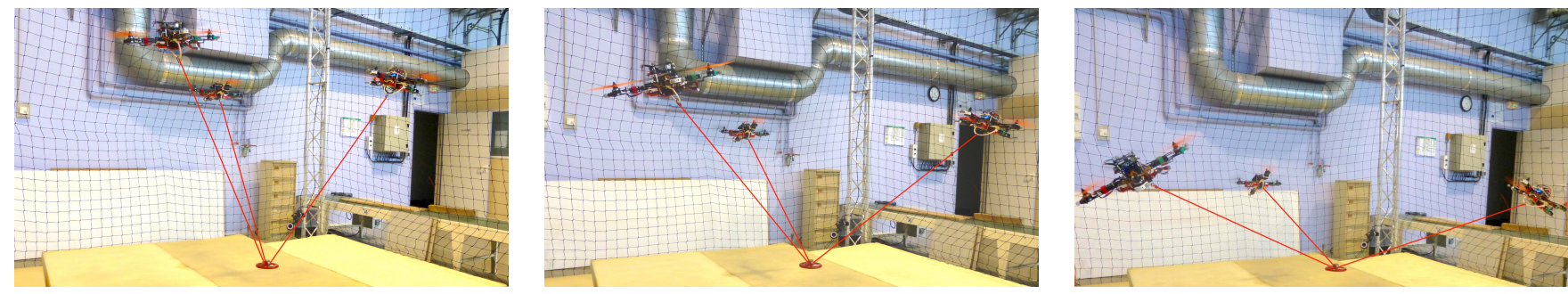

Fig. 4: The ACTS with a $1.15 \mathrm{~kg}$ payload, and inclinations of $\theta^{d}=35^{\circ}$ (left), $\theta^{d}=50^{\circ}$ (center), and $\theta^{d}=65^{\circ}$ (right).

This control law in (15) for a regular parallel robot guarantees asymptotic convergence is the absence of modelling errors [16]. As the error behaves as a second-order system $\ddot{\mathrm{e}}+k_{D} \dot{\mathbf{e}}+k_{P} \mathbf{e}=0$, the gains can be determined by choosing a cutoff frequency $\omega_{n}$ and a damping ratio $\xi$, where $k_{P}=\omega_{n}^{2}$ and $k_{D}=2 \xi \omega_{n}$. A damping ratio of $\xi=1.0$ is used to provide fast response without overshoot, and $\omega_{n}=2.5$ was chosen through simulations and experimentations.

\section{B. ATTITUDE CONTROL LOOP}

The attitude control loop is used to stabilize the orientation of $i^{t h}$ quadrotor so that the thrust vector $f_{i} \mathbf{v}_{i}$ is parallel to $\mathbf{f}_{i}^{d}$. This results in an underconstrained quadrotor rotation, with a free rotation around the axis $\mathbf{v}_{i}$. The yaw of the quadrotor is therefor fixed to $0^{\circ}$ in $\mathcal{F}_{0}$, and projected onto the plane normal to $\mathbf{f}_{i}$, to build a desired rotation matrix $\mathbf{R}^{d}$ as described in [17].

$$
\mathbf{R}_{i}^{d}=\left[\begin{array}{lll}
\frac{\left(\mathbf{z}_{i}^{d} \times \mathbf{x}_{i}^{d}\right) \times \mathbf{z}_{i}^{d}}{\left\|\mathbf{z}_{i}^{d} \times \mathbf{x}_{i}^{d}\right\|} & \frac{\mathbf{z}_{i}^{d} \times \mathbf{x}_{i}^{d}}{\left\|\mathbf{z}_{i}^{d} \times \mathbf{x}_{i}^{d}\right\|} & \mathbf{z}_{i}^{d}
\end{array}\right]
$$

where $\mathbf{x}_{i}^{d}=\left[\begin{array}{lll}1 & 0 & 0\end{array}\right]^{T}$ and $\mathbf{z}_{i}^{d}=\mathbf{f}_{i}^{d} /\left\|\mathbf{f}_{i}^{d}\right\|$, in which $\mathbf{f}_{i}^{d}$ is the output of the position control loop.

The desired rotation is expressed as a quaternion, and is used in the attitude controller described in [18]. The attitude controller acts on feedback from the inertial measurement unit (IMU), which measures the rotation expressed in $\mathcal{F}_{0}$ as the rotation matrix $\mathbf{R}_{i}$, the body fixed angular velocity $\boldsymbol{\omega}_{i}$, and the linear acceleration $\ddot{\mathbf{x}}_{i}$. This attitude controller is globally asymptotic over $\mathrm{SO}(3)$, and robust to noise for small differences between the desired and actual rotations. The response time of the attitude control loop is much faster than that of the outer control loop, so as long as there are no large discontinuities in the setpoint $\left(\mathbf{X}^{d}, \dot{\mathbf{X}}^{d}\right)$, the inner loops should be stable in experimental conditions. Indeed it is observed experimentally that apart from the time when the outer loop receives a large step input, the magnitude of the orientation error of the quadrotors is less than $0.5^{\circ}$.

\section{EXPERIMENTAL PLATFORM}

\section{A. Hardware}

The ACTS prototype is shown in Fig. 1, and is composed of three custom-built quadrotors with Lynxmotion Crazy2fly bodies, 8"x4.5" ABS propellors, 12A ESCs, MT2208 1100Kv brushless motors, and 3-cell LiPo batteries. The mass of each quadrotor is $1.05 \mathrm{~kg}$, the cables are $1.25 \mathrm{~m}$ long, and the payload is considered as a point mass. Each quadrotor uses a Pixhawk as its flight control unit (FCU) containing the IMU and flight control hardware, and has a RaspberryPi 3B (RPi) computer that communicates with the FCU using the UART pins. The quadrotors can generate up to approximately $5.0 \mathrm{~N}$ of thrust per propellor. To ensure the vector $t_{i} \mathbf{u}_{i}$ passes through $O_{i}$ regardless of the quadrotor orientation, each cable is connected to the quadrotor by means of a 2-revolute passive wrist, ensuring that the offset is less than $10 \mathrm{~mm}$.

The position feedback is given by an 8-camera Qualisys motion capture (MoCap) system, with $1 \mathrm{~mm}$ error, and a latency of $5 \mathrm{~ms}$. The centralized controller runs on a laptop with a $2.1 \mathrm{GHz}$ quad core i7 CPU, and commands are sent to the quadrotors over a $2.4 \mathrm{GHz}$ wireless LAN channel.

\section{B. Software}

The FCUs runs a real time operating system which ensures that the motor control runs at $400 \mathrm{~Hz}$, and the attitude control loop runs at $200 \mathrm{~Hz}$. The FCUs are flashed with the PX4-v2lpe open source flight stack firmware, and use the Mavlink communication protocol. The outer control loop, written in C++, runs at $50 \mathrm{~Hz}$ on the laptop and sends ROS messages with a thrust value and a desired orientation to each $\mathrm{RPi}$. The MAVROS package running on each RPi is used to convert between ROS messages and Mavlink messages. It publishes the Mavlink thrust and attitude setpoint commands as serial messages over the UART pins.

\section{CONFIGURATION EVALUATION}

Past work on trajectory design for ACTSs has focused on the generation of feasible trajectories through the property of differential flatness [6], or relies on stochastic motion planning such as RRT graphs [19]. Here, the payload trajectory $\mathbf{x}_{p}^{d}$ may be any 3D motion of the payload with a continuous second derivative, and the configuration trajectory $\mathcal{C}^{d}$ of the robot can be determined through an optimization of the wrench feasibility in task space. By considering $\mathbf{x}_{p}^{d}$ to be quasi-static, symmetry conditions set the desired configuration to be

$$
\mathcal{C}^{d}=\left[\begin{array}{llllll}
0^{\circ} & \theta^{d} & 120^{\circ} & \theta^{d} & -120^{\circ} & \theta^{d}
\end{array}\right]^{T}
$$

The capacity margin $\gamma$ is used to analyze the degree of feasibility of a configuration, and to choose the desired inclination $\theta^{d}$ giving the most robust configuration. The general methodology for calculating the capacity margin is presented in [12], and is the result of two mappings, first from thrust space $\mathcal{H}$ to tension space $\mathcal{T}$, and then to the wrench space $\mathcal{W}$ as defined in (18), and represented 


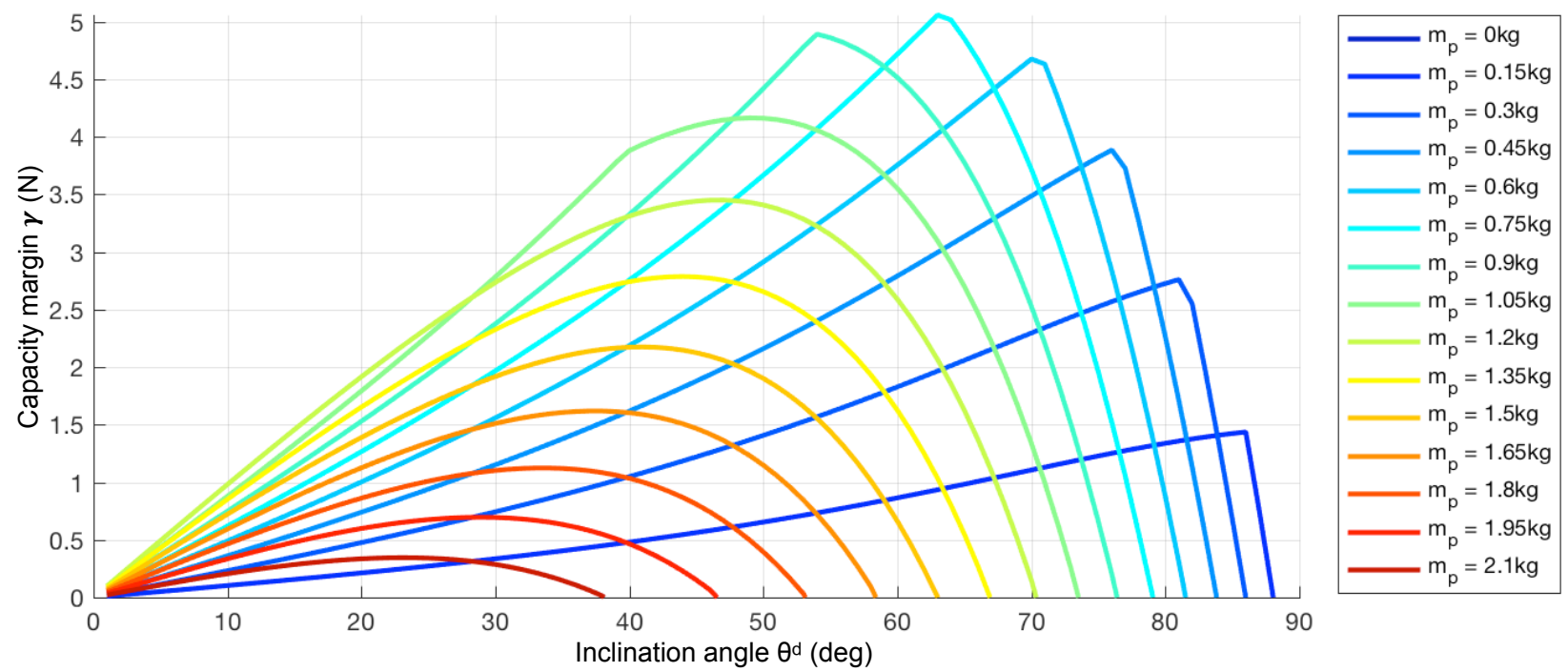

Fig. 5: Capacity margin of the symmetric ACTS as a function of the configuration and varying payload masses.


Fig. 6: Thrust, Tension, and Wrench spaces for the ACTS with a $1 \mathrm{~kg}$ payload, and $\phi=[0120,-120]^{\circ}$. The upper figure has an inclination of $\boldsymbol{\theta}=\left[\begin{array}{lll}45 & 45 & 45\end{array}\right]^{\circ}$ and the lower has $\boldsymbol{\theta}=\left[\begin{array}{lll}65 & 65 & 65\end{array}\right]^{\circ}$. The sphere is the largest that will fit inside the available wrench set, centred at the hovering wrench $\mathbf{w}=-m_{p} \mathbf{g}$, having radius $\gamma$.

in Fig. 6. It is effectively a method of representing the quadrotor actuation constraints as a task space wrench.

$$
\begin{aligned}
\mathcal{H} & =\left\{\boldsymbol{f} \in \mathbb{R}^{3}: \underline{\boldsymbol{f}} \leq \boldsymbol{f} \leq \overline{\boldsymbol{f}}\right\} \\
\mathcal{T} & =\left\{\mathbf{t} \in \mathbb{R}^{3}: \underline{\mathbf{t}} \leq \mathbf{t} \leq \overline{\mathbf{t}}\right\} \\
\mathcal{W} & =\left\{\mathbf{w} \in \mathbb{R}^{3} \mid \mathbf{w}=\sum_{j=1}^{3} \alpha_{j} \Delta t_{j} \mathbf{w}_{j}+\mathbf{W} \underline{\mathbf{t}}, \quad 0 \leq \alpha_{j} \leq 1\right\}
\end{aligned}
$$

where $\mathbf{w}$ is a feasible wrench that the ACTS can exert, $\Delta t_{j}=$ $\bar{t}_{j}-\underline{t}_{j}$, and $\mathbf{w}_{j}$ is the $j^{t h}$ column of $\mathbf{W}$.

The quadrotors each generate a maximum thrust of $20 \mathrm{~N}$ and the maximum thrust of the $\mathrm{i}^{\text {th }}$ quadrotor, $\bar{f}_{i}$, is limited to $90 \%$ of this value to prevent thrust saturation from interfering with the generation of moments required to control the orientation. As the cable is very light, a minimum tension $\mathrm{t}=0.1 \mathrm{~N}$ is sufficient to ensure geometric form closure. The upper bound $\bar{t}_{i}$ of the $i^{t h}$ cable tension $t_{i}$ is expressed as:

$$
\bar{t}_{i}=m_{i} \mathbf{g}^{T} \mathbf{u}_{i}+\sqrt{\bar{f}_{i}^{2}+m_{i}^{2} g^{2}\left(u_{i z}^{2}-1\right)}
$$

The available wrench set $\mathcal{W}$ is then found as the convex hull of the mapping of the tension space vertices to wrench space, and the capacity margin is found as the minimum signed distance between the wrench $-m_{p} \mathbf{g}$ and the boundary of the AWS zonotope, with a positive sign indicating inclusion. In Fig. 5. the capacity margin is plotted as a function of $\theta$ for a range of payload masses. Figures 5 and 6 are used to determine a configuration for the ACTS. It can be seen that a positive (although low) capacity margin exists until the cables are nearly vertical, while wider configuration have a larger capacity margin, yet the maximum value of $\theta^{d}$ for which the capacity margin is positive decreases as the payload mass increases. The flatter AWS at large $\theta^{d}$ makes the payload resistive to lateral forces, while the tall AWS at lower $\theta^{d}$ result is a larger lift capacity. Lower and upper limits are imposed such that $35^{\circ} \leq \theta^{d} \leq 70^{\circ}$ to avoid collisions between quadrotors, and singularities respectively.

\section{RESULTS}

Three experimental tests are presented in this paper. Section VI-A demonstrates the accuracy of the trajectory tracking for the position and configuration. Section VI-B shows the effect of varying configurations on the tracking error, and Sec. VI-C demonstrates the effect of increased $\ddot{\mathbf{x}}_{p}^{d}$ on the performance.

\section{A. Trajectory Tracking}

To test the ACTS's tracking performance, the trajectory seen in Fig. 7 is performed with a $1.15 \mathrm{~kg}$ mass. The desired 


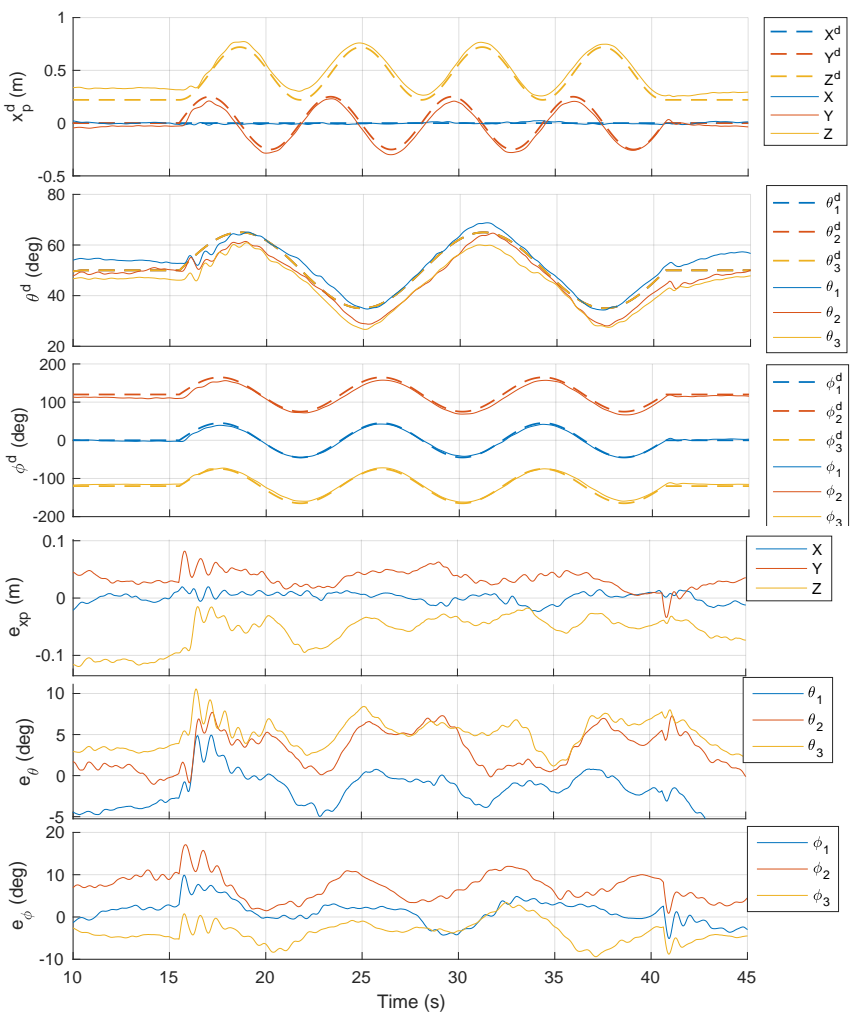

Fig. 7: Trajectory tracking with a $1.15 \mathrm{~kg}$ payload. The top three graphs showing the desired (dashed lines) and actual (solid lines) values of the position $\mathbf{x}_{p}$, inclination angles $\boldsymbol{\theta}$, and azimuth angles $\phi$ respectively. The bottom three graphs show the evolution of the error in $\mathbf{x}_{p}, \boldsymbol{\theta}$, and $\phi$.

inclination $\theta^{d}$ is bounded between $35^{\circ}$ and $65^{\circ}$, less than the maximum feasible configuration of $\theta^{d}=71^{\circ}$ (from Fig. 5). The payload had an root mean square error of $8.0 \mathrm{~cm}$, including a constant offset of $\approx 5 \mathrm{~cm}$ along the $z_{0}$ axis. The tracking error for the configuration is about $\pm 5^{\circ}$ for $\theta$ and $\pm 10^{\circ}$ for $\phi$, including a noticeable amount of biased error. The constant component of the error in the configuration varying between each quadrotor is indicative of an inaccurate mapping between the thrust command and the actual thrust produced by the quadrotor. Furthermore it was found after experimentations that a measurement bias of $+1 \%$ was present in the mass measurements of the payload and quadrotors, thus overestimating the thrust required to compensate for gravitational and inertial effects.

\section{B. Configuration Limits}

To test that a positive capacity margin $\gamma$ accurately indicates the controllability of the ACTS, payloads of $m_{p}=1.15 \mathrm{~kg}, 1.35 \mathrm{~kg}, 1.65 \mathrm{~kg}, 1.85 \mathrm{~kg}, 2.05 \mathrm{~kg}$, and $2.15 \mathrm{~kg}$ were tested, following a simply quasi-static trajectory $\left(\left\|\ddot{\mathbf{x}}_{p}^{d}\right\|<0.1 \mathrm{~ms}^{-2}\right)$. The desired configuration parameter $\theta^{d}$ was varied, beginning at $35^{\circ}$ and increasing at a rate of $1.5^{\circ} \mathrm{s}$. Figure 8 shows the mean inclination $\theta$ of the three cables, and the magnitude of the payload position error $\mathbf{e}_{p}$ as functions of time.

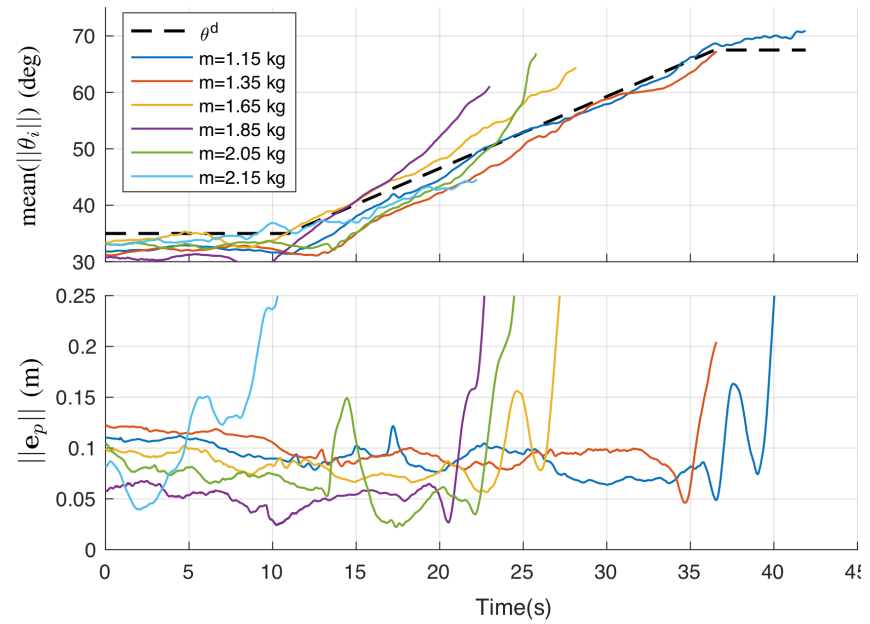

Fig. 8: Plots of the mean inclination angle of the ACTS configuration (top), and of the tracking error of the payload position (bottom) for six different payload masses

TABLE I: Comparison of the mean $\theta$ when the ACTS crashed (error diverges $>0.15 \mathrm{~m}$ ) to the predicted configuration limit of $\theta(\gamma=0 \mathrm{~N})$

\begin{tabular}{lc|cccccc}
\hline \hline Mass & $(\mathrm{kg})$ & 1.15 & 1.35 & 1.65 & 1.85 & 2.05 & 2.15 \\
\hline $\bar{\gamma}$ & $(N)$ & 3.7 & 2.8 & 1.6 & 1.0 & 0.5 & 0.25 \\
$\theta(\gamma=0)$ & $(\mathrm{deg})$ & 71 & 67 & 58 & 51 & 40 & 34 \\
$\theta_{\text {crash }}$ & $(\mathrm{deg})$ & 70 & 65 & 60 & 55 & 50 & 35 \\
Error & $(\mathrm{deg})$ & 1 & 2 & -2 & -4 & 10 & -1 \\
\hline \hline
\end{tabular}

Table I compares the mean inclination angle $\theta$ of the ACTS where the error diverges in Fig. 8 to the upper value of $\theta$ when $\gamma=0 \mathrm{~N}$ in Fig. 5. It shows a clear correspondence between the unstable configuration predicted by the wrench analysis of the symmetric ACTS, and the configuration where the error diverges. It can be seen that despite a wide range of $\gamma$ over each test, there is no significant change in accuracy until the system becomes wrench infeasible. The difference in accuracy between trials can be attributed partially due to imprecisely calibrated masses, and partially due to variances in the system between tests. Changes in ambient lighting, and local wifi usage affected the performance of the ACTS.

\section{Dynamic Trajectories}

To investigate the performance under dynamic conditions, $\mathcal{C}^{d}$ is fixed, and the payload trajectory in (20) is imposed, where the desired payload acceleration increases in magnitude by $\left\|\ddot{\mathbf{x}}_{p}^{d}\right\|=t^{2} / 3600 \mathrm{~ms}^{-2}$.

$$
\mathbf{x}_{p}^{d}(t)=\left[\begin{array}{c}
0.25 \sin \left(t^{2} / 30\right) \\
0 \\
-0.25 \cos \left(t^{2} / 30\right)+0.75
\end{array}\right] \mathrm{m}, t \geq 0 \mathrm{~s}
$$

Figure 9 shows the magnitude of the payload tracking error and the mean thrust of the quadrotors for tests at three configurations, $\theta^{d}=35^{\circ}, \theta^{d}=50^{\circ}, \theta^{d}=65^{\circ}$. The $\left\|\mathbf{e}_{p}\right\|$ increases around $t=50 \mathrm{~s}$ for the $\theta^{d}=65^{\circ}$ configuration $\left(\left\|\ddot{\mathbf{x}}_{p}^{d}\right\| \approx 0.7 \mathrm{~ms}^{-2}\right)$, and at $60 s\left(\left\|\ddot{\mathbf{x}}_{p}^{d}\right\| \approx 1.0 \mathrm{~ms}^{-2}\right)$ for 


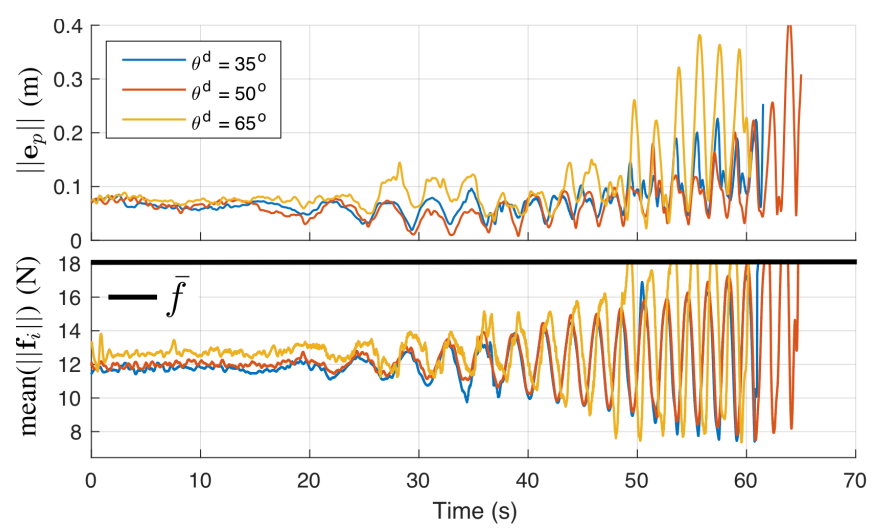

Fig. 9: Plot of the payload position tracking error (top), and the mean quadrotor thrust (bottom) for a $0.65 \mathrm{~kg}$ payload at three different configurations.

the narrower configurations, despite the value of $\phi(\gamma=0)$ being $80^{\circ}$. This spike in error happens at the first saturation $\mathbf{f}^{d}$, indicating that for accelerations of $\ddot{\mathbf{x}}_{p}^{d}=0.7 \mathrm{~ms}^{-2}$ the static wrench analysis is no longer sufficient. It can be seen also however, that choosing an appropriate configuration can greatly increase the achievable accelerations of the ACTS.

\section{CONCLUSIONS AND FUTURE WORK}

The controller proposed in this paper was able to successfully track payload position and configuration trajectories when the ACTS is wrench feasible. It is shown that the capacity margin index gives an accurate estimation of the region of feasibility of the ACTS for quasi-static trajectories, but must be adapted to account for quadrotor dynamics which become significant even at low accelerations. The capacity margin is shown to serve as an indicator of controllability and not of accuracy, and it is recommended to extend it to dynamic cases for configuration planning purposes to maximize the acceleration capabilities of the ACTS. The controller is sensitive to modelling errors, present in the weight measurements of the quadrotors and payload. A proposed solution to be investigated in the future is the inclusion of adaptive controllers such as those studied in [20] and [21] for CDPRs and in [22] for a single quadrotor with an uncertain slung mass load.

Further work is anticipated on this subject in several areas in order to develop a more externally useful system. These include modifying the available wrench set calculations to account for the system dynamics, extending the ACTS to a $6 \mathrm{DOF}$ platform, and the introduction of actuation redundancy through more cables. It is also interesting for deployment in an external environment to move to a decentralized control scheme where the configuration state of the robot is measured through onboard sensors or observers.

\section{REFERENCES}

[1] V. Kumar and N. Michael, "Opportunities and challenges with autonomous micro aerial vehicles," The International Journal of Robotics Research, vol. 31, no. 11, pp. 1279-1291, August 2012.

[2] F. Ruggiero, V. Lippiello, and A. Ollero, "Aerial manipulation: A literature review," IEEE ROBOTICS AND AUTOMATION LETTERS, vol. 3, no. 3, pp. 1957-1964, July 2018.
[3] H. Khamseh, F. Janabi-Sharifi, and A. Abdessameud, "Aerial manipulation-a literature survey," Robotics and Autonomous Systems, vol. 107, pp. $221-235,2018$.

[4] Y. Alothman, M. Guo, and D. Gu, "Using iterative lqr to control two quadrotors transporting a cable-suspended load," International Federation of Automatic Control IFAC-papers online, vol. 50, pp. 4324-4329, July 2017.

[5] J. Fink, N. Michael, S. Kim, and V. Kumar, "Planning and control for cooperative manipulation and transportation with aerial robots," The International Journal of Robotics Research, vol. 30, no. 3, pp. 324-334, 2011.

[6] K. Sreenath and V. Kumar, "Dynamics, control and planning for cooperative manipulation of payloads suspended by cables from multiple quadrotor robots," in Robotics: Science and Systems, June 2013.

[7] T. Lee, K. Sreenath, and V. Kumar, "Geometric control of cooperating multiple quadrotor uavs with a suspended payload," in Proceedings of the 52nd IEEE Conference on Decision and Control. IEEE, December 2013.

[8] H. Rastgoftar, E. Taheri, A. Ghasemi, E. Atkins, and G. A, "Continuum deformation of a multi-quadcopter system in a payload delivery mission," in The International Federation of Automatic Control, July 2017.

[9] A. Tagliabue, M. Kamel, S. Verling, R. Siegwart, and J. Nieto, "Collaborative transportation using mavs via passive force control," in Proceedings of the International Conference on Robotics and Automation. IEEE, 2017.

[10] M. Tognon, C. Gabellieri, L. Pallottino, and A. Franchi, "Aerial comanipulation with cables: The role of internal force for equilibria, stability, and passivity," in IEEE Robotics and Automation Letters, IEEE, Ed., vol. 3, no. 3, July 2018.

[11] C. Masone, H. Bülthoff, and P. Stegagno, "Cooperative transportation of a payload using quadrotors: a reconfigurable cable-driven parallel robot," in International Conference on Intelligent Robots and Systems. IEEE, October 2016.

[12] J. Erskine, A. Chriette, and S. Caro, "Wrench capability analysis of aerial cable towed systems," in Proceedings of the ASME 2018 International Design Engineering Technical Conferences \& Computers and Information in Engineering Conference, Quebec City, Canada, August 26-29 2018.

[13] S. Bouchard, C. Gosselin, and B. Moore, "On the ability of a cabledriven robot to generate a prescibed set of wrenches," ASME Journal of Mechanisms and Robotics, vol. 2, no. 1, 2010.

[14] A. L. Cruz Ruiz, S. Caro, P. Cardou, and F. Guay, "Arachnis: Analysis of robots actuated by cables with handy and neat interface software," Mechanisms and Machine Science, vol. 32, pp. 293-305, 2015.

[15] L. Gagliardini, S. Caro, M. Gouttefarde, and A. Girin, "Discrete reconfiguration planning for cable-driven parallel robots," Mechanism and Machine Theory, vol. 100, pp. 313-337, 2016.

[16] F. Paccot, N. Andreff, and P. Martinet, "A review on the dynamic control of parallel kinematic machines: Theory and experiments," The International Journal of Robotics Research, vol. 28, no. 3, pp. 395416, March 2009

[17] T. Lee, M. Leoky, and N. H. McClamroch, "Geometric tracking control of a quadrotor uav on se(3)," in Proceedings of the 49th IEEE Conference on Decision and Control, IEEE, Ed. IEEE, December 2010.

[18] D. Brescianini, M. Hehn, and R. D'Andrea, "Nonlinear quadrocopter attitude control technical report," Institute for Dynamic Systems and Control (IDSC) ETH Zurich, Tech. Rep., October 2013.

[19] M. Manubens, D. Devaurs, L. Ros, and J. Cortes, "Motion planning for 6-d manipulation with aerial towed-cable systems." in Robotics: Science and Systems, June 2013, p. 8.

[20] E. Picard, S. Caro, F. Plestan, and F. Claveau, "Control solution for a cable-driven parallel robot with highly variable payload," in Proceedings of the ASME 2018 International Design Engineering Technical Conferences and Computers and Information in Engineering Conference, Quebec City, Canada, August 26-29 2018.

[21] J. Lamaury, M. Gouttefarde, A. Chemori, and P. E. Herve, "Dual-space adaptive control of redundantly actuated cable-driven parallel robots," in IEEE International Conference on Intelligent Robots and Systems, 2013, pp. pp. 4879-4886.

[22] A. Dai, T. Lee, and D. Bernstein, "Adaptive control of a quadrotor uav transporting a cable-suspended load with unknown mass,", in Proceedings of the 53rd IEEE Conference on Decision and Control, Los Angeles, CA, 2014, pp. pp. 6149-6154. 